\title{
A case of actinomycosis of the middle ear
}

\author{
R Ologunde, J Doshi
}

Birmingham Heartlands Hospital, Bordesley Green East, Birmingham, B9 5SS, UK

\section{Introduction}

Actinomycosis infection of the ear is a rare slowly progressive granulomatous and suppurative disease, with less than 50 cases reported in the literature worldwide. We report a case of middle ear actinomycosis and discuss the importance of histopathology guided diagnosis.

\section{Case}

A 66-year-old lady presented to the otology clinic with a 5-year history of chronic left sided otorrhoea. Past medical history included a left parotidectomy and subsequent radiotherapy, 24 years prior, for a tumour of unknown histology. Computed Tomography scanning revealed extensive opacification of the external canal and left mastoid cavity in addition to bony erosion of the external canal and posterior aspect of the middle ear cavity but an intact ossicular chain. Magnetic Resonance Imaging confirmed the diffuse enhancement of the changes in the left mastoid and middle ear cavity in keeping with an inflammatory aetiology or a diffuse destructive soft tissue process.

A variety of topical steroid and antibiotic treatments were unsuccessful over a period of years before she was referral to the otology clinic. Histopathological examination of an ear biopsy suggested actinomycosis but the organism was not identified on culture. The patient was commenced on a 12-month course of oral amoxicillin. At 12months, the ear was dry and this clinical improvement was reflected in repeat MR imaging.

\section{Imaging}

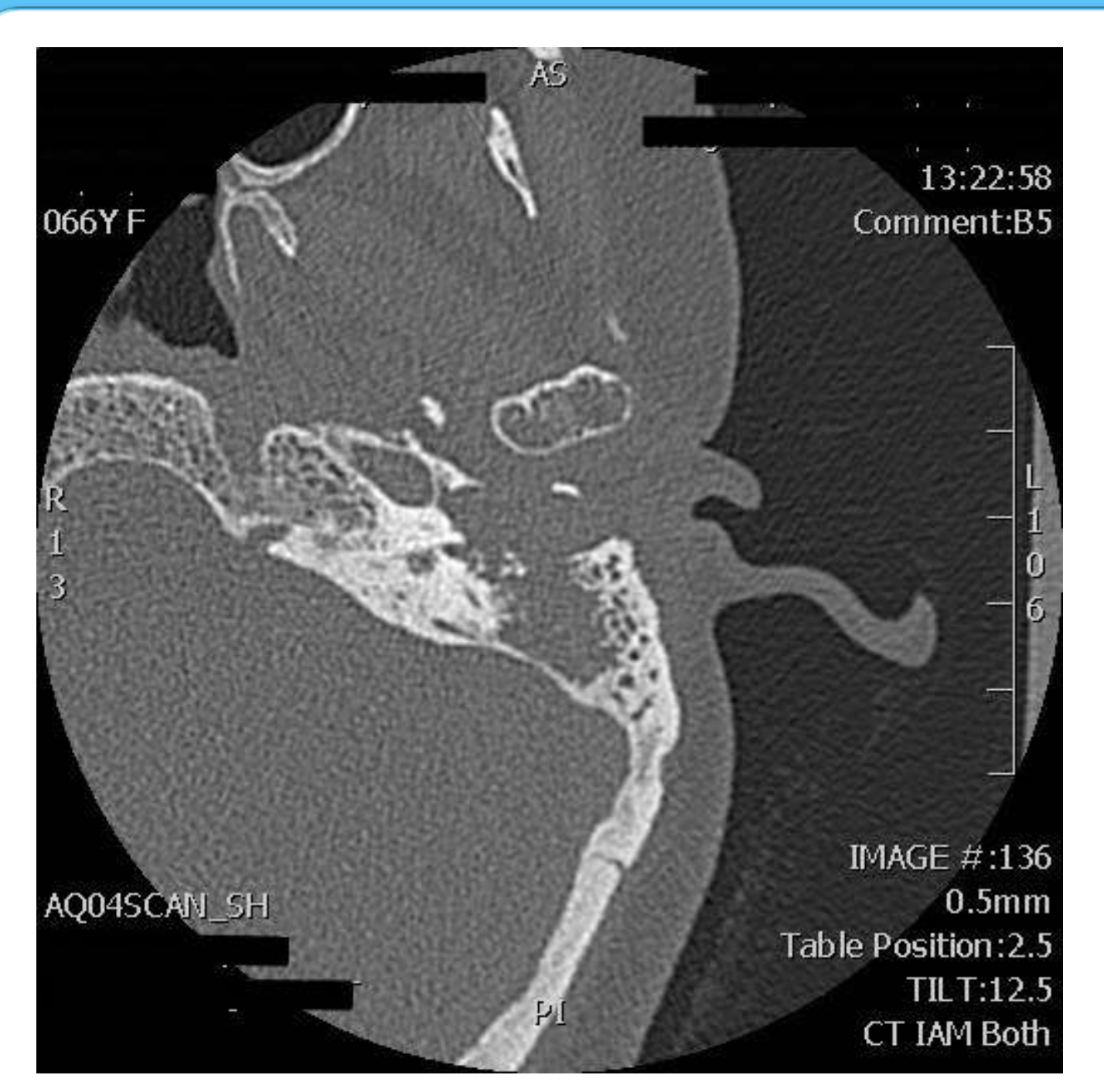

\section{Literature Review}

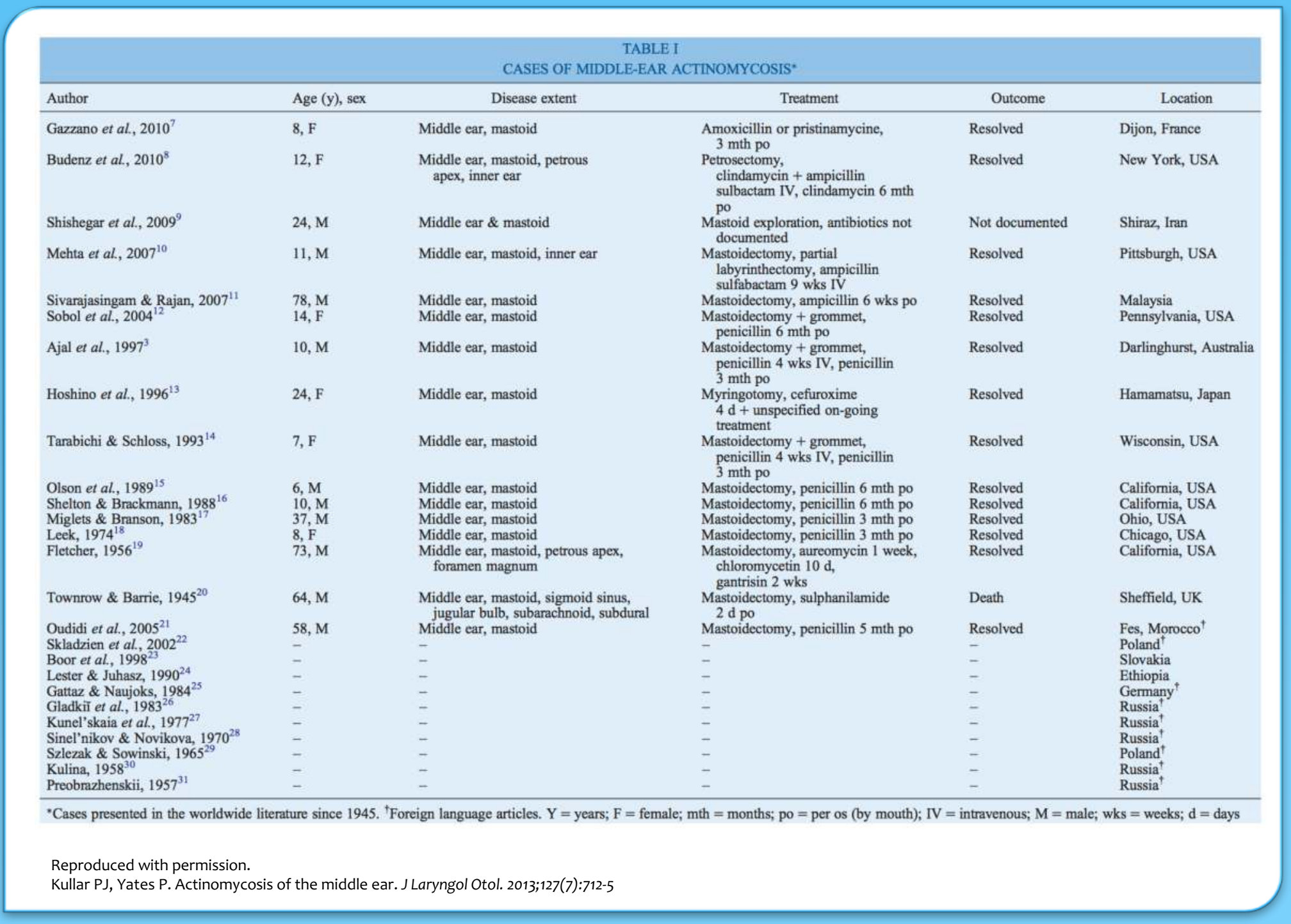

\section{Conclusion}

The differential diagnosis in this case included osteoradionecrosis (secondary to previous radiotherapy) and recurrence of the parotid tumour. The actinomycosis colony was an entirely incident finding on biopsy. Diagnosis of this condition is made difficult because of the non-specific nature of the clinical signs and the difficulty in culturing the organism. 